\title{
modélisation en centrifugeuse de problèmes d'interaction sol-structure
}

\section{centrifuge modelling of soil-structure interaction problems}

\author{
H.L. JESSBERGER \\ Université de la Ruhr, Bochum (RFA)*
}

Rev. Franç. Géotech. n 48, pp. 49-54 (juillet 1989)

\section{Résumé}

Ce rapport fait une revue complète des problèmes d'interaction sol-structure traités par modèle réduit en centrifugeuse, à l'exception des problèmes dynamiques. Les références d'études publiées sont données et brièvement discutées en s'appuyant sur des exemples d'applications concrètes. Les problèmes pratiques et les développements nécessaires pour les futures recherches en centrifugeuse sont abordés.

\section{Abstract}

This paper is a comprehensive review of centrifuge modelling of soil-structure interaction problems, excepting dynamic phenomena. Literature references are briefly discussed based on examples of engineering applications. Engineering problems and necessary developments for future centrifuge investigations are explained. 


\section{INTRODUCTION}

En mai 1987 , se tenait à Paris une conférence internationale sur l'interaction sol-structure. Les problèmes de remblais, de fondations superficielles et profondes, d'ouvrages souterrains, d'excavations et d'ouvrages de soutènement $y$ furent discutés. Nombreuses sont les communications présentées qui peuvent inspirer les chercheurs géotechniciens pour des travaux de modélisation en centrifugeuse. Il est évident qu'avec un développement continuel en géotechnique, de nouveaux problèmes d'interaction sol-structure se feront jour et devront être résolus. Certains aspects des apports présents et futurs de la modélisation en centrifugeuse seront discutés ci-après. Chaque partie traite successivement de la description du problème dans sa relation avec la situation réelle, de la contribution de la modélisation en centrifugeuse et examine les développements futurs.

\section{OUVRAGES SOUTERRAINS ET STRUCTURES ENTERRÉES}

\subsection{Les problèmes pratiques}

Les ouvrages souterrains ont une importance croissante en génie civil. Les traits principaux relatifs aux problèmes d'interaction sol-structure pour les ouvrages souterrains sont portés sur la figure 1.

Une couche de sol en état d'équilibre naturel est perturbée par une excavation de géométrie donnée, par exemple un tunnel circulaire qui représentera aussi d'autres types de cavités comme des puits ou des cavernes. L'excavation provoque des changements dans la distribution des contraintes dans le sol, ce jusqu'à ce qu'un nouvel état d'équilibre soit atteint. Les éléments de revêtement agissent comme facteur stabilisateur. D'autres processus mécaniques peuvent avoir une influence comme des variations de la nappe; les efforts apportés par les semelles des bâtiments voisins, la circulation des véhicules, ou les déformations de la couche elle-même, provoquée par exemple par leur affaissement en zone minière ou des mouvements tectoniques.

Certaines de ces questions sont d'un intérêt majeur pour les applications pratiques comme:

- l'évolution des déformations autour des tunnels, revêtus ou non, jusqu'à la rupture;

- l'interaction périphérique entre le revêtement et le sol environnant;

- l'interaction longitudinale entre le revêtement du tunnel, des canalisations et le sol, en relation avec le processus d'excavation, les changements de couches de sol, le tassement, les mouvements de failles, etc.

\subsection{Apports de la modélisation en centrifugeuse}

La plupart des études en centrifugeuse au cours de la dernière décennie se sont focalisées sur des modèles bidimensionnels. Les sols utilisés ont été des sols pulvérulents et des sols cohérents, en général des sables standards et du kaolin. La préparation des
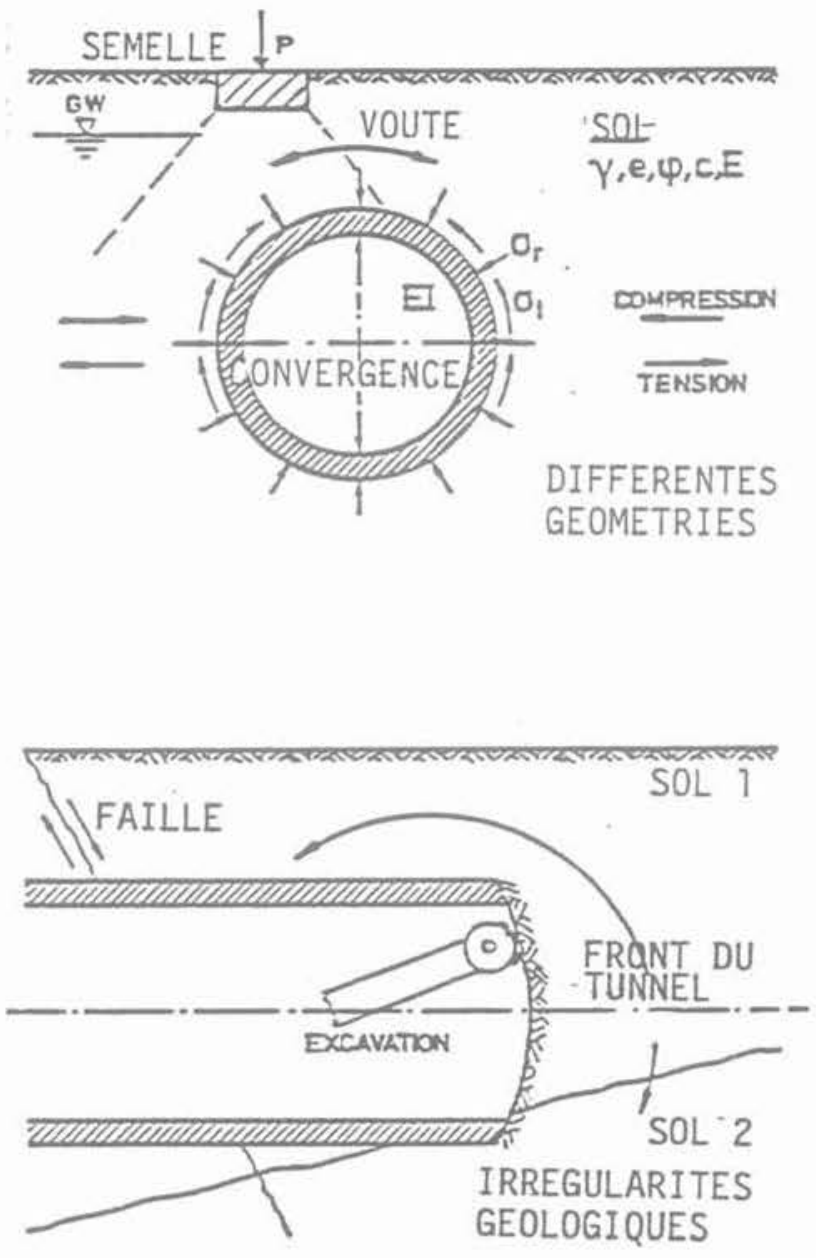

Fig. 1. - Interaction sol-structure

pour des ouvrages souterrains.

Fig. 1. - Soil structure interaction of underground structures.

modèles de sable s'effectue usuellement en enfouissant la structure dans le sable déposé en pluie à 1 $\mathrm{g}$; le modèle est alors accéléré dans la centrifugeuse jusqu'au niveau de contraintes requis puis le processus physique qui doit être étudié est recréé.

Les modèles d'argile sont préparés par consolidation d'une boue jusqu'à obtention d'un certain profil de consolidation. Ceci peut être obtenu par chargement à l'aide d'un piston ou par la méthode du gradient hydraulique hors de la centrifugeuse et sur la machine, grâce à l'accélération centrifuge. Après consolidation, un certain volume d'argile est excavé pour déposer la structure souterraine. La mise en rotation du modèle complet dans la centrifugeuse conduit au profil final de consolidation.

Les études en centrifugeuse sur les problèmes de stabilité en section courante et en front de tunnels, ainsi que celles sur les puits ont été résumées par MAIR et al., (1984). POTTS (1976) a traité du comportement de tunnels dans les sables. De nombreuses expériences ont été faites en assurant la tenue des excavations non revêtues par une membrane en caoutchouc avec une pression interne d'air. A un niveau de $\mathrm{g}$ donné, la pression interne était réduite par paliers et les déformations et, les mécanismes de 
rupture étaient observés. POTTS a utilisé un revêtement d'aluminium équipé de jauges pour détecter l'évolution des déformations et la distribution des contraintes dans le revêtement sous la pression des terres.

Des études sur des canalisations rigides et souples enterrées dans des tranchées ont été effectuées par JAMES et LARSSON, (1975), VALSANGKAR et BRITTO (1977). Ces tuyaux étaient équipés de jauges pour mesurer les déformations. TROTT et al. (1984) ont démontré de façon convaincante quel usage peut être fait des modèles réduits en centrifugeuse pour analyser le comportement des ouvrages prototypes. Les distributions de déformations, les charges limites et la déformée finale des tuyaux sous l'effet de chargements par bandes sur différentes zones de la surface du sol montrent un bon accord entre modèle et prototype.

Des modèles tridimensionnels de canalisations enterrées ont été étudiés par KUSAKABE (1984) à propos des mouvements de sol, engendrés par l'excavation, à proximité, de tranchées susceptibles de provoquer la rupture des canalisations enterrées. La comparaison entre le mécanisme de rupture supposé et celui observé sur les modèles centrifugés ont conduit à penser que ledit mécanisme pouvait être employé pour les applications.

Le comportement d'un tunnel soumis à un mouvement tectonique a été examiné par BURRIDGE (1984), en vue d'obtenir des informations sur l'interaction entre le tunnel et le sol, les niveaux de contrainte que l'on peut atteindre dans le tunnel et la longueur de tunnel affectée par ces mouvements. Une technique spéciale a été utilisée pour produire le mécanisme de faille dans le modèle. Des jauges d'extensométrie ont été employées pour déceler les déformations du tunnel représenté par un tube en aluminium.

Les communications présentées au congrès Centrifugeuse 88 concernent la pression s'exerçant sur des conduites enterrées rigides (TOHDA et al., 1988) et l'effet de charges statiques et roulantes sur des buses flexibles (CRAIG et MOKRANI, 1988). Ces deux articles donnent de bons exemples d'instrumentation et de techniques de chargement de modèle élaborées. Ces développements améliorent la qualité des informations sur le comportement mécanique obtenues sur chaque modèle, par suite, le nombre d'essais nécessaires pour vérifier les résultats peut être réduit.

Les exemples précédents et d'autres qui peuvent être trouvés dans les références bibliographiques montrent que les progrès dans les apports de la modélisation en centrifugeuse aux applications pratiques portent sur les problèmes de détermination de conditions de stabilité en milieu pulvérulent ou cohérent. Des solutions ont été établies qui permettent de calculer les efforts sur le tunnel selon la résistance au cisaillement mobilisée dans les terrains de couverture, résistance qui est une fonction des déformations dans le sol et par conséquent des déformations admises au front du tunnel et autour du revêtement. De même, des distributions de contrainte dans les revêtements et les conduites ont été déterminées pour différentes conditions aux limites comme les charges apportées par des semelles, des essieux roulants, par des excavations ou par l'extraction de palplanches à proximité de conduites. Les résultats ont donné une meilleure connaissance des transferts d'efforts du sol aux structures enterrées et ont permis d'améliorer les critères de dimensionnement.

\subsection{Développements futurs}

Des développements dans la technique des modèles sont nécessaires pour étendre lapplication des études en centrifugeuse. Certains peuvent être résumés ainsi:

- les études bidimensionnelles devraient porter sur d'autres formes de sections. Les interactions mutuelles entre structures enterrées voisines devraient être considérées

- la modélisation tridimensionnelle d'effets de voûte et la détermination des tassements de surface près du front de tunnel sont des problèmes importants. Les phénomènes d'interaction entre le sol et des structures plus complexes comme les zones de raccordement de tunnels ou des cavernes, devraient être étudiés en centrifugeuse;

- les équipements d'essai devraient être développés pour permettre d'aborder les phénomènes d'interaction qui sont affectés principalement par les processus d'excavation, car les problèmes sur site ne peuvent pas être traités par les critères ordinaires de construction. La modélisation en centrifugeuse peut aider à comprendre de telles situations et aider le concepteur;

- la technique de mise en place des structures dans le sol du modèle devait être améliorée pour réduire linfluence de la procédure de construction du modèle. De même, les techniques de modélisation devraient être développées pour prendre en compte les anomalies géologiques susceptibles de causer des problèmes d'instabilité pendant la construction des ouvrages souterrains.

\section{OUVRAGES DE SOUTÈNEMENT}

\subsection{Les problèmes pratiques}

Les excavations profondes à proximité de bâtiments ou de constructions existantes ont stimulé de rapides progrès dans la conception des ouvrages de soutènement.

La figure 2 montre quelques questions intéressant le projeteur. Deux principes de construction peuvent être distingués: d'abord les murs, comme les murs en béton qui sont construits isolément et remblayés après exécution: en second lieu, les écrans comme les parois moulées mis en place dans le sol, puis excavés d'un côté.

Différents systèmes de sollicitation agissant sur le soutènement pendant les phases de construction doivent être pris en considération pour le dimensionnement. 

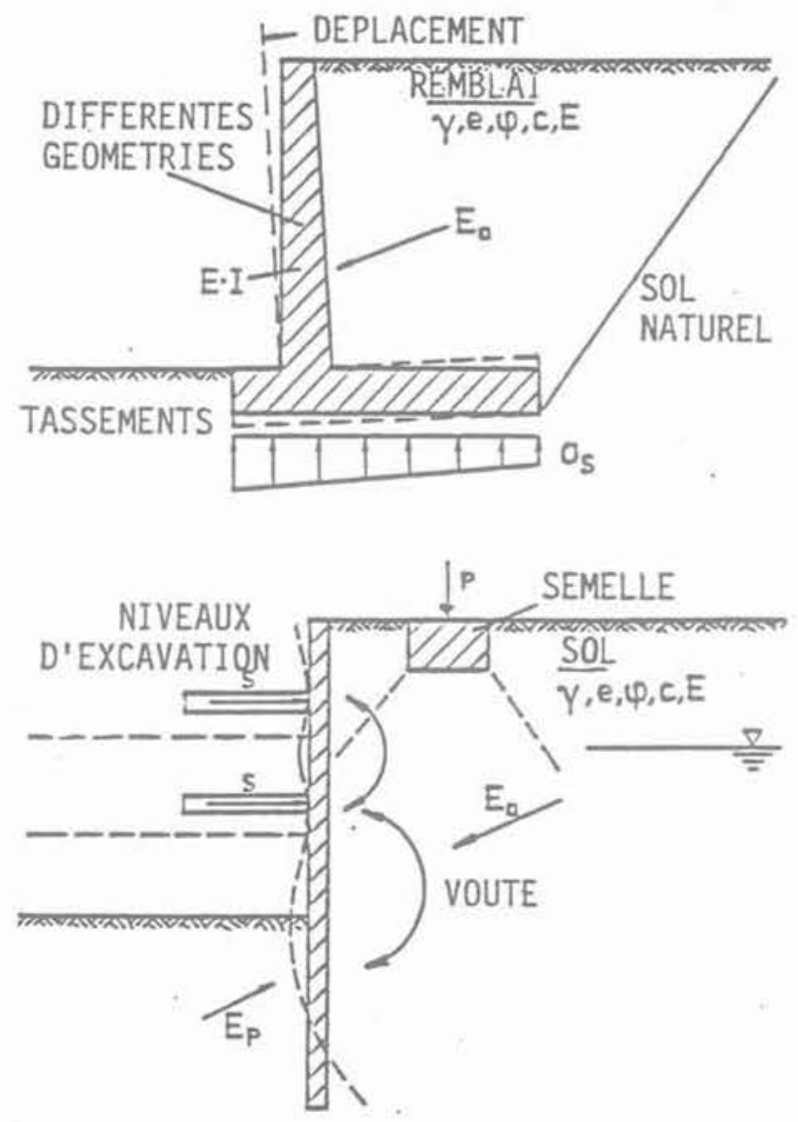

Fig. 2. - Ouvrages de soutènement.

Fig. 2. - Retaining structures.

Bien que des méthodes analytiques fondées sur les théories de pressions limites soient disponibles, nombre de questions sont encore non résolues. En particulier, les variations dans la distribution de la pression des terres et les effets de voûte, provoqués par différentes rigidités et conditions d'appui sont des facteurs relativement méconnus. Des problèmes peuvent survenir quand les conditions de déformation plane ne sont pas satisfaites, parce que les conditions aux limites requièrent une analyse tridimensionnelle pour le dimensionnement du mur.

\subsection{Apports de la modélisation en centrifugeuse}

Dans la dernière décennie, la modélisation en centrifugeuse a porté principalement sur des études en déformation plane. LYNDON et PEAESON (1984) ont étudié le comportement d'un mur rigide et le développement des pressions des terres dues à une excavation par phases devant le mur jusqu'à la rupture. Les résultats sur les distributions de pression se comparaient bien avec les théories admises. BOLTON et MAK (1984) étudièrent le problème d'une semelle filante superficielle posée sur un massif de sable contenu par un mur de soutènement en forme de L. Ces deux recherches mentionnées ici servaient d'études paramétriques sur des structures idéalisées.
Un modèle tridimensionnel d'un ouvrage prototype constitué par une paroi non renforcée, conçue comme une coque mince pour soutenir une excavation circulaire fut étudié par RIGDEN et ROWE (1975). Les panneaux étaient préparés en microbéton. Les expériences ont porté sur différents chargements et différentes conditions de sol.

Les communications à Centrifugeuse 88 couvrent des études paramétriques sur des configurations idéalisées pour observer les mouvements du sol et du mur, les distributions des pressions des terres et les mécanismes de rupture, ainsi qu'un cas concret pour analyser la ruine d'un mur de quai. BOLTON et al. (1988) ont rendu compte d'un ensemble important d'essais en centrifugeuse pour étudier le comportement d'un écran dans une argile surconsolidée. L'excavation en pied de mur était simulée par la vidange d'une enveloppe de caoutchouc contenant une solution de chlorure de zinc destinée à maintenir l'équilibre des contraintes pendant la phase d'accélération du modèle jusqu'au niveau de $\mathrm{g}$ requis. Les études faites par SCHERBINA (1988) ont porté sur un mur rigide tournant autour d'une articulation à sa base. Le mur était instrumenté à l'aide de cellules de pression à différents niveaux. Les distributions de pression des terres associées à un mouvement cyclique du mur autour d'un angle initial sont présentées, ainsi que celles correspondant à des déplacements unidirectionnels du mur. ZHU et YI (1988) ont montré l'emploi d'un modèle réduit centrifugé pour faire l'analyse a posteriori de la rupture d'un mur de quai intervenue lors d'opérations de dragage. Des campagnes de reconnaissance détaillées effectuées après l'accident ont indiqué que la résistance au cisaillement retenue pour le dimensionnement était supérieure à la résistance mobilisable effectivement dans le sol sous la fondation du quai. Les modèles centrifugés ont confirmé le mode de rupture de l'ouvrage prototype. Des analyses de stabilité complémentaires ont montré que le facteur de sécurité était inférieur à la valeur critique.

Les exemples cités montrent que la modélisation en centrifugeuse de problèmes de murs de soutènement peut apporter en pratique des résultats appréciables pour la conception, et cela particulièrement pour des sols cohérents.

\subsection{Développements futurs}

Il semble important de souligner pour les recherches futures que la modélisation en centrifugeuse de problèmes de soutènements devrait porter sur des structures plus complexes. Les situations standard sont traitées en principe de manière efficace par des critères de dimensionnement simples. Les connaissances font par contre toujours défaut sur les questions suivantes: - les variations des distributions de contrainte dans les écrans souples supportés à différents niveaux, par exemple par des ancrages. Les effets de voûte devraient être étudiés;

- l'influence des différentes phases d'excavation sur la distribution des contraintes dans de tels systèmes; - le comportement de l'ouvrage pendant le remblaiement et le compactage; 
- l'influence de conditions de sols difficiles, en particulier avec des zones de sol mou sous les fondations de murs de soutènement;

- le comportement de différents types de murs cellulaires;

- des problèmes tridimensionnels comme aux angles des murs.

\section{SUPERSTRUCTURES}

\subsection{Les problèmes pratiques}

Les problèmes principaux pour le comportement des superstructures sont: le tassement d'ensemble, les tassements différentiels, la distribution des contraintes dans la fondation et les effets des tassements différentiels sur la distribution des contraintes dans la superstructure. Une situation typique est celle présentée sur la figure 3 , une couche d'argile non uniforme consolide sous la charge de la construction et provoque des tassements différentiels.

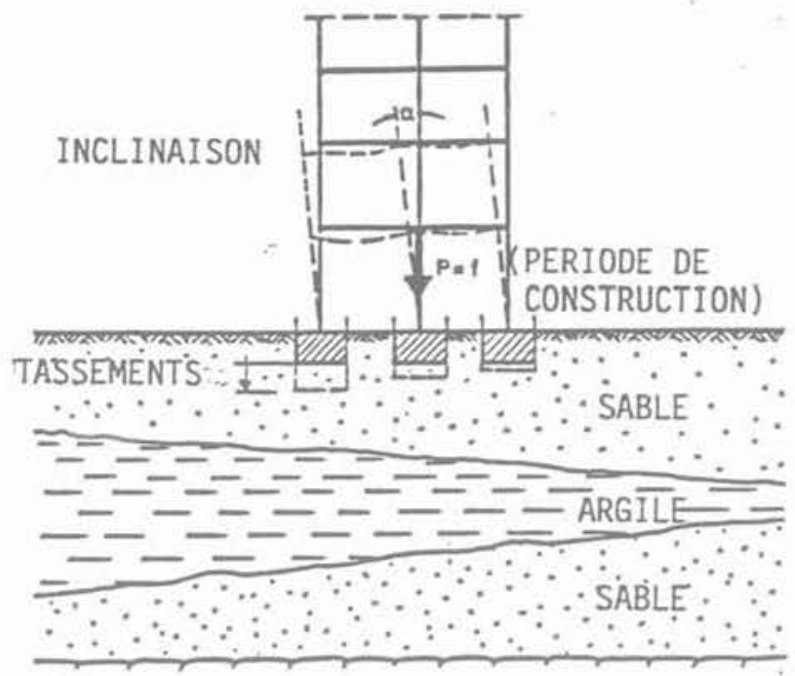

Fig. 3. - Tassements différentiels d'une superstructure.

Fig. 3. - Differential settlements of a superstructure.

\subsection{Apports de la modélisation en centrifugeuse}

Peu d'études sur ce sujet ont été publiées à ce jour, si l'on fait exception des études de capacité portante avec semelles rigides. Quelques informations sont fournies par ROWE (1981) concernant les platesformes pétrolières offshore. Des résultats d'essais ont été donnés décrivant le comportement d'une grande fondation reposant sur une couche d'argile uniforme et soumise à un chargement horizontal cyclique. Des résultats d'essais sur un problème similaire ont été publiés par PREVOST et al. (1981). Des fondations de plates-formes offshore gravitaires établies sur un limon sableux et argileux ont été soumises à des chargements cycliques axiaux, inclinés centrés et excen- trés, et les tassements ont été évalués en fonction du temps. Le comportement d'un réservoir de stockage de pétrole, fondé sur de l'argile molle a été présenté par KIM et al. (1983). Des profils de tassement sous le réservoir ont été donnés en fonction du temps.

XIE (1988) a décrit l'emploi d'essais en centrifugeuse pour déterminer les contraintes dans la structure d'une centrale électrique construite sur une fondation non uniforme. Le sol sous l'ouvrage prototype était composé de couches de basalte d'épaisseur et de rigidité variables. Ces couches étaient représentées par de l'époxy et d'autres matériaux de substitution. Le bâtiment de la centrale était lui aussi réalisé en époxy. Le modèle entier était chauffé dans un conteneur et accéléré à $100 \mathrm{~g}$. Après refroidissement, les contraintes prisonnières dans le modèle ont été estimées par les méthodes de photo-élasticité.

Des résultats intéressants ont été présentés par ABGHARI et al. (1988). Les auteurs ont décrit des modèles réduits en centrifugeuse conçus pour étudier l'instabilité par renversement de tours élancées, avec une référence particulière à la tour de Pise. Ils ont montré que les observations faites avec quelques essais en centrifugeuse n'auraient jamais pu être obtenues avec une autre méthode, ou seulement avec des essais sur site extrêmement onéreux.

Fondamentalement, il y a un large éventail de recherches possibles concernant les problèmes d'interaction entre sol et superstructures. Des difficultés de construction du modèle peuvent se faire jour quand il faut représenter précisément et les superstructures et leurs fondations. De plus, la modélisation de la durée de la phase de construction peut être particulièrement importante quand la consolidation et la diffusion des pressions interstitielles affectent le comportement de louvrage prototype.

\subsection{Développements futurs}

Des essais sur les superstructures devraient trouver plus de place dans la modélisation en centrifugeuse et pourraient être reliés aux problèmes suivants:

- tassement d'ensemble et différentiels des superstructures provoqués par un sol non homogène, comportant essentiellement des sols cohérents;

- interaction entre fondations de tailles différentes et à différents niveaux de chargement;

- examen de la phase de construction, en relation avec les problèmes de consolidation et de stabilité; - influence de nouvelles constructions à proximité de bâtiments existants.

\section{CONCLUSIONS}

Dans de nombreux cas, il est important pour le projeteur de décrire précisément l'interaction entre le sol qui est chargé par la structure, et la structure, qui est influencée par la réaction du sol. Dans la première approche, des méthodes analytiques ou numériques 
sont employées. Mais les résultats des calculs doivent être interprétés eu égard aux propriétés des matériaux et conditions géométriques particulières du site. Dans ces situations, lavantage de la modélisation en centrifugeuse vient de la possibilité d'étudier le système sol-structure dans son entier et dès lors d'étudier directement le problème d'interaction.

Lors du congrès Centrifugeuse 88,7 communications se rattachaient au thème interaction sol-structure.

Plusieurs communications ont donné des exemples probants d'emploi avec succès de modèles centrifugés. Il conviendrait de chercher à développer les techniques de construction et d'intervention sur les modèles, par exemple, pour simuler en rotation les procédures d'excavation et d'insertion. D'autres développements devraient porter vers la représentation de différentes conditions de sol incluant des hétérogénéités.

\section{REMERCIEMENTS}

L'auteur remercie le Dr. ULRICH GÜTTLER pour l'aide qu'il a apportée dans la préparation de ce rapport.

\section{BIBLIOGRAPHIE}

ABGHARI A., CHENEY J.A., KUTTER B.L. (1988), Leaning instability of tall structures. Centrifuge' 88 , pp. $435-442$.

BOLTON M.D., MAK K.W. (1984), The application of centrifuge models in the study of an interaction problem. Proceedings of a Symposium on the Application of Centrifuge Modelling to Geotechnical Design, Manchester.

BOLTON M.D., POWRIE W., STEWART D.I. (1988). The design of deep in situ walls. Centrifuge'88, pp. 405-414.

BURRIDGE P.B. (1984), Behaviour of a tunnel during a rapid earthquake faulting episode. Proceedings of the Symposium on Recent Advantge in Geotechnical Centrifuge Modelling, University of California, Davis.

GRAIG W.H., MOKRANI A. (1988), The effect of static line and rolling axle loads on flexible culverts buried in granular soil. Centrifuge' 88 , pp. 385-394.

JAMES R.G., LARSSON H. (1975), Centrifugal model tests of buried rigid pipes. Research Report, University of Cambridge, Department of Engineering, Cambridge.

JESSBERGER H.L., GÜTTLER U., STOFFERS U. (1987). Centrifuge model investigations on the interaction of shallow tunnels and surrounding soil. International Conference on Soil Structure Interactions, Paris.
KIM Y.S., SHEN C.K., BANG S. (1983), Oil storage tank foundation on soft clay. VIII European Conference on Soil Mechanics and Foundation Engineering, Helsinki.

KUSAKABE O (1984), Centrifuge model tests on the influence of an axisymmetric excavation on buried pipes. Proceedings of International Symposium on Geotechnical Centrifuge Model Testing, Tokyo.

LYNDON W., PEARSON R.A. (1984), Pressure distribution on a rigid retaining wall in cohesionless material. Proceedings of a Symposium on the Application of Centrifuge Modelling to Geotechnical Design, Manchester.

MAIR R.J., PHILLIPS R., SCHOFIELDS A.N., TAY. LOR R.N. (1984), Application of centrifuge modelling to the design of tunnels and excava. tions in soft clay. Proceedings of a Symposium on the Application of Centrifuge Modelling to Geotechnical Design, Manchester.

POTTS D.N. (1976), Behaviour of lined and unlined tunnels in sand. Doctoral Dissertation. University of Cambridge, Department of Engineering. Cambridge.

PREVOST J.H., CUNY B., SCOTT R.F. (1981), Offshore gravity structures: Centrifugal Modeling. Journal of the Geotechnical Engineering Division, Vol. 107, n GT2.

RIGDEN W.J., ROWE P.W. (1975), Model performance of an unreinforced diaphragm wall. Diaphragm walls and anchorages, Institution of Civil Engineers, London.

ROWE P.W. (1981), Use of large centrifugal models for offshore and nearshore works. Symposium on Geotechnical Aspects of Coastal and Offshore Structures, Bangkok.

SCHERBINA V.I. (1988), Earth pressure studies on retaining walls by centrifugal modelling. Centrifuge' 88 , p. 421-426.

TOHDA J., MIKASA M., HACHIYA M. (1988), Pressure acting on underground rigid pipes centrifuge model tests and FEM analysis - . Centrifuge'88, pp. 395-402.

TROTT J.J., TAYLOR R.N., SIMONS I.F, (1984), Test to validate centrifuge modelling of flexible pipes. Proceedings of a Symposium on the Application of Centrifuge Modelling to Geotechnical Design, Manchester.

VALSANGKAR A.J., BRITTO A.N. (1977), The vali. dity of ring compression theory in the design of flexible buried pipes. Research Report, University of Cambridge, Department of Engineering, Cambridge.

XIE G. (1988), Centrifuge modelling on experimen. tal stress analusis for the structures built on nonuniform foundation. Centrifuge'88, pp. 429-434.

ZHU W., YI J. (1988), Application of centrifuge modelling to study a failed quay wall. Centrifuge' 88 , pp. $415-420$. 